This document is confidential and is proprietary to the American Chemical Society and its authors. Do not copy or disclose without written permission. If you have received this item in error, notify the sender and delete all copies.

\title{
Evaluating the Performance of Water Models with Host- Guest Force Fields in Binding Enthalpy Calculations for Cucurbit[7]uril-Guest Systems
}

\begin{tabular}{|c|c|}
\hline Journal: & The Journal of Physical Chemistry \\
\hline Manuscript ID & jp-2020-11383e.R2 \\
\hline Manuscript Type: & Article \\
\hline $\begin{array}{r}\text { Date Submitted by the } \\
\text { Author: }\end{array}$ & $n / a$ \\
\hline Complete List of Authors: & $\begin{array}{l}\text { Cinaroglu, Suleyman Selim; University of Oxford, Department of } \\
\text { Biochemistry } \\
\text { Biggin, Philip; University of Oxford, Biochemistry }\end{array}$ \\
\hline
\end{tabular}

\section{SCHOLARONE \\ Manuscripts}




\title{
Evaluating the Performance of Water Models with Host-Guest Force Fields in Binding Enthalpy Calculations for Cucurbit[7]uril-Guest Systems
}

\author{
Süleyman Selim Çınaroğlu and Philip C. Biggin* \\ Department of Biochemistry, University of Oxford, South Parks Road, Oxford, OX1 3QU, UK \\ *To whom correspondence should be addressed. \\ Philip.biggin@bioch.ox.ac.uk
}

ORCID 0000-0001-5100-8836 


\begin{abstract}
Computational prediction of thermodynamic components with computational methods has become increasingly routine in computer-aided drug design. Although, there has been significant recent effort and improvements in the calculation of free energy, the prediction of enthalpy (and entropy) remains under-explored. Furthermore, there has been relatively little work reported so far that attempts to comparatively assess how well different force-fields and water models perform in conjunction with each other. Here, we report a comprehensive assessment of force fields and water models using host-guest systems that mimic many features of protein-ligand systems. These systems are computationally inexpensive possible because of their small size compared to protein-ligand systems. We present absolute enthalpy calculations using the multi-box approach on a set of 25 cucurbit[7]uril-guest pairs. Eight water models were considered (TIP3P, TIP4P, TIP4P-Ew, SPC, SPC/E, OPC, TIP5P, Bind3P), along with five force fields commonly used in the literature (GAFFv1, GAFFv2, CGenFF, Parsley and SwissParam). We observe that host-guest binding enthalpies are strongly sensitive to the selection of force-field and water model. In terms of water models, we find that TIP3P, and its derivative Bind3P are the best performing models for this particular host-guest system. The performance is generally better for aliphatic compounds than aromatic ones, suggesting aromaticity remains a difficult property to include accurately in these simple force fields.
\end{abstract}




\section{INTRODUCTION}

The sophistication and capability of computational methods to understand biomolecules and their interactions is increasing rapidly. ${ }^{1-2}$ Predicting the binding affinities of candidate molecules for a specific target is a prime goal within computational drug discovery. ${ }^{3-4}$ Computational studies can provide not only the binding free energy but also entropic and enthalpic components. ${ }^{5-6}$ Prediction of the thermodynamic components using molecular simulations is crucial to get insight into the structural elaboration and physical interactions of molecular recognition. Getting the thermodynamic subcomponents of the free energy reveals the molecular driving forces at work and hence should help guide the design of high-affinity binders for the target. Furthermore, an understanding of these contributions at the atomic level, may help us to better comprehend the phenomenon of entropy-enthalpy compensation. ${ }^{7-10}$

Host-guest systems are promising for studying calorimetric calculations because of their size, enabling long time-scale molecular dynamics (MD) simulations. ${ }^{11-12}$ They mimic many features of larger protein-ligand systems that require more computational resource. ${ }^{13-14}$ Importantly, the binding affinities of host-guest systems are generally in the same range as protein-ligand systems. 15-16 Thus, their small size, simple structure, and predictable protonation states make them ideal miniature models of molecular recognition. ${ }^{15}$ Their simple structures overcome setup problems in MD simulations, and their small sizes make it possible to reach numerical convergence faster than protein-ligand systems in explicit-solvent for binding free energy calculations. ${ }^{17-18}$

Host-guest systems as tiny models are alternative for informative testing and improving forcefields before using them in protein-ligand systems. ${ }^{19}$ It may be complicated to choose which force field to use in MD simulations for protein-ligand systems, as new options are continually being offered. Simulators generally prefer to select the most cited or popular force field in their case, but the ability of all force-fields to replicate experimental data is controversial. ${ }^{20-21}$ Protein-ligand systems as a benchmark would perhaps seem ideal to test force-fields. However, many factors, such as simulation setups, long-timescale protein dynamics, $\mathrm{pH}$ effects and so on, make these tests complicated. Host-guest systems offer a benchmark series that overcome many of these difficulties for the assessment studies of force-fields. Furthermore, they may help not only to select appropriate force-fields for MD simulation but also to optimize force-fields for a specific case. ${ }^{20-21}$

In addition to general force-fields, the force-field parameters of water are crucial factors in molecular simulations using explicit water models. ${ }^{22}$ Although water is a tiny and simple molecule, 
replicating its properties with MD simulations is still challenging. ${ }^{23-26}$ The choice of water model significantly affects the simulation results since all water models represent water and its interactions according to various approaches or paradigms. ${ }^{23-24,27}$ Rigid water models with a fixed charge distribution are popular and commonly used, but their ability to accurately predict entropic and enthalpic components still needs to be improved. Thus, assessment studies of the water models have been required to determine which model is capable of producing structural and dynamic properties of the water. ${ }^{28}$

A number of studies have reported how the choice of water model and force-fields affects the accuracy of MD simulations for the prediction of thermodynamic properties. 6, 19, 29-31 Fenley et al. 31 compared TIP3P to TIP4P-Ew water models in binding enthalpy calculations for 8 cucurbit[7]uril-guest pairs, showing that theTIP3P water model yields more accurate results than TIP4P-Ew. Subsequently, Gao et al. ${ }^{29}$ investigated the salt dependency on the binding enthalpy across four water models, TIP3P, SPC/E, TIP4P-Ew, and OPC, with the choice of parameters for sodium and chloride using a neutral host-guest pair. Henriksen and Gilson ${ }^{19}$ examined the influence of four water models (TIP3P, TIP4P-Ew, SPC/E, and OPC), ion parameters, partial charge assignment methods, and the cyclodextrin force field on thermodynamic entities. Yin et al. 30 introduced a new TIP3P derivative called Bind3P, using the sensitivity analysis approach. They evaluated Bind3P by comparing it with the TIP3P, TIP4P-Ew, OPC, and TIP4P-D in thermodynamics calculations. In the most recent work, TIP3P and Bind3P were compared in a representative set of organic micropollutants binding $\beta$-cyclodextrin. Another recent work reports a comprehensive study of the diffusivity of $\alpha-, \beta$-, $\gamma$-cyclodextrin in aqueous solutions using TIP3P, SPC/E,TIP4P/2005 and Bind3P water models. ${ }^{32}$ Thus a significant amount of work has already been done in this area. However, a comparison of widely used methods for general force-field parametrization has not yet been presented. In this work, we use the direct method with the multibox approach to determine the binding enthalpy and comparatively assess eight rigid models for water along with five different parametrization methods for the host and guests. The results further highlight the sensitivity to force-field parameter choice in estimating these values.

\section{METHODS}

\section{Host-Guest Systems and Force Fields}

We used 25 host-guest pairs obtained from various publications to calculate the binding enthalpy. 33-38 We selected ITC experiments without salts or buffers since they may yield significant errors 
relative to experimental data when used to compute host-guest binding enthalpies. ${ }^{29}$ Structures are represented in Figure 1 and are listed in Table 1. Also, the acyclic cucurbituril-derived TrimerTrip clip molecule with a guest molecule (heptane-1,7-diamine) was used for further investigations (Figure 5A). ${ }^{39}$ We performed MD simulations using five different force field (CGenFF ${ }^{40-41}$, GAFFv1 ${ }^{42}$, GAFFv2 ${ }^{42}$, Parsley ${ }^{43}$, and SwissParam ${ }^{44}$ ) and eight different water models (OPC ${ }^{45}$, SPC ${ }^{46}$, SPC/E ${ }^{47}$, TIP3P ${ }^{48}$, TIP4P ${ }^{48}$, TIP4P-Ew ${ }^{49}$, TIP5P ${ }^{50}$, Bind3P ${ }^{30}$ ). We only used the TIP3P water model for the TrimerTrip simulations. Strictly speaking, these water models are also force-fields, but the term "water model" is commonly used in the literature and we adhere to that convention hereafter.

GAFFv1 and GAFFv2 parameters and partial atomic charges obtained with the AM1-BCC method 51-52 were generated by AMBER's antechamber tool ${ }^{50}$ while CGenFF parameters and atomic charges ${ }^{40-41}$ were obtained using the CGenFF web server. ${ }^{53}$ SwissParam ${ }^{54}$ topologies and parameters with MMFF charges were generated by using the SwissParam server. ${ }^{55}$ In this work we were mainly focused on "out of the box" performance, but others have reported for GAFF at least that consideration of different charge models (RESP and AM1-BCC) appears to make little difference. 19 Parameter and coordinate files are provided for each force field as supplementary information. 

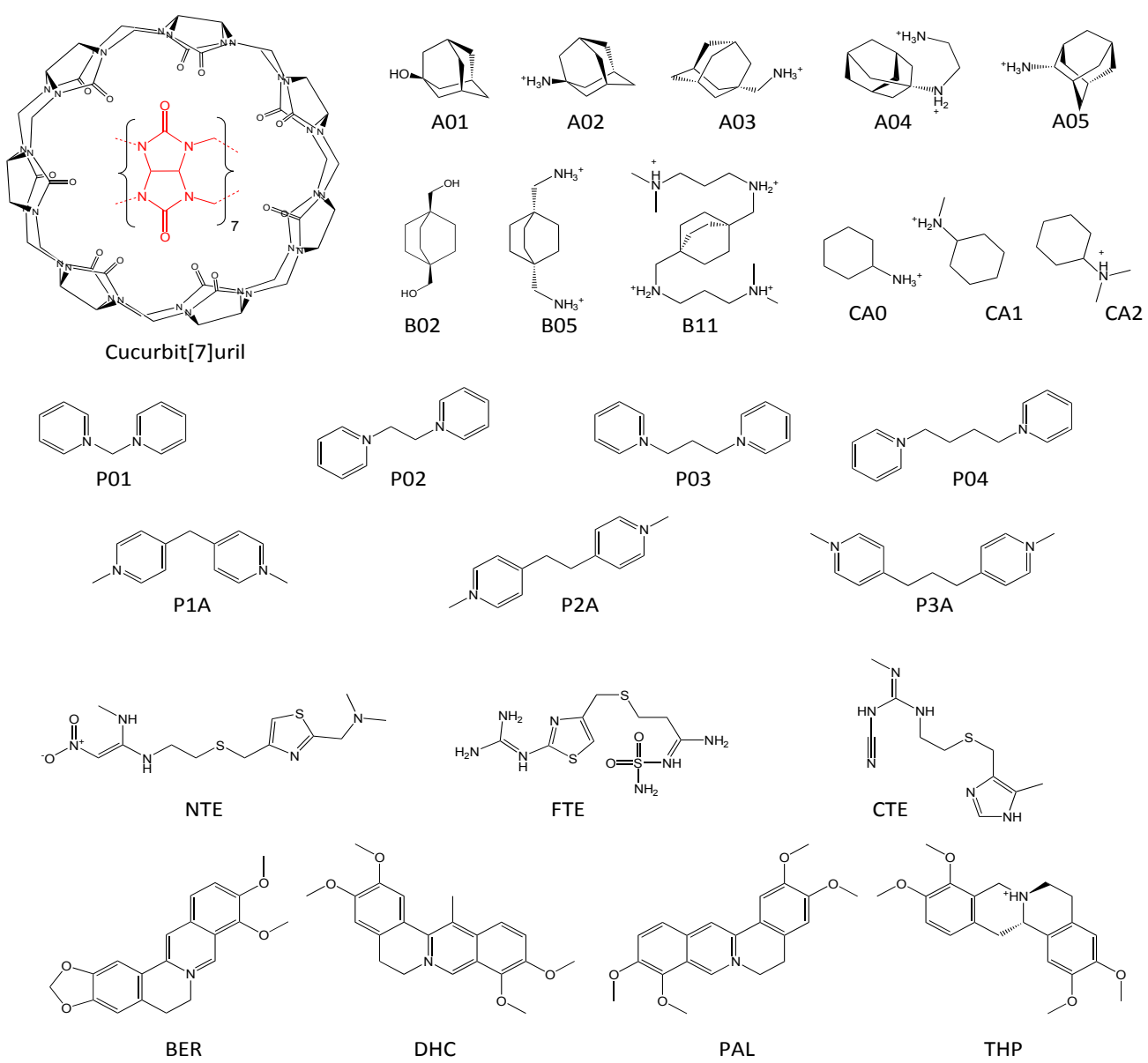

DHC

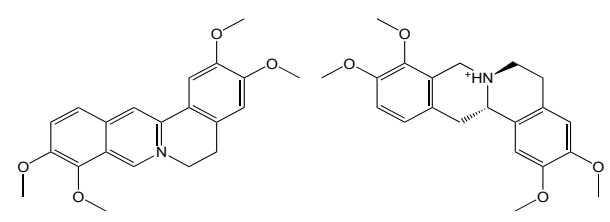

PAL

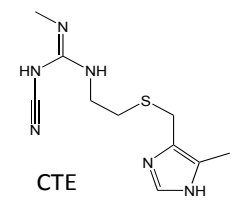

Figure 1. Chemical structures of the host (cucurbit[7]uril) and guest molecules 
Table 1: Guest molecules with their experimental binding affinities including entropic and enthalpic components.

\begin{tabular}{|c|c|c|c|c|}
\hline Guest & $\Delta \mathbf{G}$ & $-\mathrm{T} \Delta \mathrm{S}$ & $\Delta \mathrm{H}$ & Ref. \\
\hline A01 & $-14.1 \pm 0.2$ & $4.9 \pm 0.4$ & $-19.0 \pm 0.4$ & 33 \\
\hline A02 & $-19.4 \pm 0.1$ & $-0.1 \pm 0.5$ & $-19.3 \pm 0.4$ & 33 \\
\hline A03 & -20.3 & 1.7 & $-21.9 \pm 0.4$ & 33 \\
\hline A04 & -21.5 & -1.4 & $-20.1 \pm 0.4$ & 33 \\
\hline A05 & $-19.1 \pm 0.2$ & $0.4 \pm 0.5$ & $-19.5 \pm 0.4$ & 33 \\
\hline B02 & $-13.4 \pm 0.1$ & $2.4 \pm 0.2$ & $-15.8 \pm 0.2$ & 33 \\
\hline B05 & $-19.5 \pm 0.2$ & $-3.9 \pm 0.5$ & $-15.6 \pm 0.4$ & 33 \\
\hline B11 & $-20.6 \pm 0.4$ & $-4.3 \pm 0.5$ & $-16.3 \pm 0.4$ & 33 \\
\hline BER & $-12.95 \pm 0.36$ & $-3.87 \pm 0.28$ & $-9.08 \pm 0.5$ & 37 \\
\hline CAO & $-10.79 \pm 0.22$ & $-0.88 \pm 0.09$ & $-9.91 \pm 0.2$ & 36 \\
\hline CA1 & $-9.17 \pm 0.16$ & $1.65 \pm 0.07$ & $-10.82 \pm 0.1$ & 36 \\
\hline CA2 & $-8.76 \pm 0.16$ & $2.82 \pm 0.07$ & $-11.58 \pm 0.1$ & 36 \\
\hline CTE & $-5.69 \pm 0.16$ & $7.57 \pm 0.01$ & $-13.2 \pm 4.0$ & 34 \\
\hline $\mathrm{DHC}$ & $-8.55 \pm 0.28$ & $-0.43 \pm 0.14$ & $-8.12 \pm 0.24$ & 38 \\
\hline FTE & $-6.40 \pm 0.04$ & $4.68 \pm 0.01$ & $-11.1 \pm 0.4$ & 34 \\
\hline NTE & $-5.64 \pm 0.09$ & $9.96 \pm 0.01$ & $-15.6 \pm 2.2$ & 34 \\
\hline P01 & $-8.43 \pm 0.02$ & $-4.45 \pm 0.01$ & $-3.98 \pm 0.02$ & 35 \\
\hline P02 & $-8.15 \pm 0.11$ & $-1.69 \pm 0.04$ & $-6.45 \pm 0.10$ & 35 \\
\hline P03 & $-8.86 \pm 0.04$ & $-3.70 \pm 0.0$ & $-5.16 \pm 0.04$ & 35 \\
\hline P04 & $-8.83 \pm 0.15$ & $-4.29 \pm 0.05$ & $-4.55 \pm 0.15$ & 35 \\
\hline $\mathrm{P} 1 \mathrm{~A}$ & $-8.28 \pm 0.06$ & $-1.30 \pm 0.05$ & $-6.98 \pm 0.03$ & 35 \\
\hline P2A & $-8.98 \pm 0.03$ & $-2.39 \pm 0.01$ & $-6.58 \pm 0.02$ & 35 \\
\hline P3A & $-8.75 \pm 0.10$ & $-1.67 \pm 0.06$ & $-7.08 \pm 0.08$ & 35 \\
\hline PAL & $-9.83 \pm 0.64$ & $-1.00 \pm 0.43$ & $-8.84 \pm 0.64$ & 38 \\
\hline THP & $-6.88 \pm 0.54$ & $0.43 \pm 0.36$ & $-7.31 \pm 0.41$ & 38 \\
\hline
\end{tabular}

All values are in $\mathrm{kcal} / \mathrm{mol}$ and to the number of significant digits originally reported. Uncertainties are given as standard deviations of the mean.

\section{Molecular Dynamics Simulations}

All simulations were performed using the Gromacs v2019 software package. ${ }^{56-58}$ A periodic cubic water box was used for all systems with 1500 water molecules while 3000 water molecules for TrimerTrip simulations. A 3-step steepest descent energy minimization with a maximum force of $10 \mathrm{~kJ} / \mathrm{mol} / \mathrm{nm}$ was applied to all systems. ${ }^{59}$ In the first step, all heavy atoms were implemented position restraints with the harmonic potential at a force constant of $1000 \mathrm{~kJ} /\left(\mathrm{mol} \mathrm{nm}^{2}\right)$, after those restraints on water molecules were removed, and then the final step removed all position restraints. All systems were equilibrated with a 5 ns NVT and NPT simulations. The V-rescale 60 and Parrinello-Rahman ${ }^{61}$ algorithms were used for temperature and pressure coupling to control the temperature at $300 \mathrm{~K}$ and an isotropic pressure at 1.0 bar, respectively. Unbonded interactions were calculated up to a cut-off of $1.0 \mathrm{~nm}$ with a potential-shift modifier for GAFFv1, GAFFv2, and Parsley simulations while the calculation was done using a cut-off of $1.2 \mathrm{~nm}$ with a force-switching for CGenFF and SwissParam simulations. A dispersion correction was only applied to energy and 
pressure for GAFFv1, GAFFv2, and Parsley. All H-bond lengths were constrained with a LINear Constraint Solver (LINCS) algorithm. ${ }^{62}$ Coulomb interactions were evaluated with the Fast smooth Particle-Mesh Ewald (SPME) electrostatics method with an initial short-range cutoff of 1.0 (GAFFv1, GAFFv2, and Parsley) and 1.2 (CGenFF and SwissParam) nm. ${ }^{63}$ The leap-frog algorithm was used to integrate the equations of motion for $100 \mathrm{~ns}$ MD simulation runs with the periodic boundary conditions. An integration time step was set to 2 fs for all simulations.

\section{Binding Enthalpy Calculations}

The binding enthalpy $(\Delta H)$ is calculated by Equation 1, where $\langle\mathrm{E}\rangle_{\text {complex }},\langle\mathrm{E}\rangle_{\text {water }}$, $\langle\mathrm{E}\rangle_{\text {host }}$, and $\langle\mathrm{E}\rangle_{\text {guest }}$ are the averaged potential energies of the system from four separate simulations of the host-guest complex, water, host, and guest, respectively. This multi-box approach was implemented by Fenley et al. ${ }^{31}$ In this approach, the number of solvent molecules should exactly balance the stoichiometry of the simulations.

$$
\Delta H=\langle\mathrm{E}\rangle_{\text {complex }}+\langle\mathrm{E}\rangle_{\text {water }}-\langle\mathrm{E}\rangle_{\text {host }}-\langle\mathrm{E}\rangle_{\text {guest }}
$$

Bonded and unbonded states simulations of host-guest complex should have an equal number of water molecules. If ions, such as sodium and chloride, are present in the solution, then these ions must similarly be balanced between these states. Note that the pressure-volume contribution is negligible for the binding enthalpy.

\section{Error Analysis}

The standard error of the mean (SEM) is calculated using the blocking method without running time-consuming additional simulations. ${ }^{64-66}$ The pyblock python module ${ }^{67}$ was used to estimate the SEM for time-correlated data series. It implements a reblocking analysis to remove serial correlation from a simulation data and obtain an improved SEM. The blocking method iteratively calculates averages of the simulation data series into consecutively larger blocks and calculates the SEM for each block. The experimental SEM listed here were obtained by dividing observed standard deviations by the square root of the number of replicates for a fair comparison. 


\section{RESULTS \& DISCUSSION}

Numerous examples of binding affinity calculations using MD simulations are available in the literature. 11-12, 18-19, 29 Here, this study provides additional insight for the comparison of the forcefields and water models combinations in the binding enthalpy calculations. We report a comprehensive comparison of the various combinations tested in this work, showing which combination of parameters has good agreement with experiment for the enthalpy calculation. For this purpose, 2080 independent MD simulations were performed on 25 host-guest systems having experimental data available (Table 1), using the direct, multi-box approach for the binding enthalpy calculations from the difference in mean potential energies. 
Table 2. Statistics between the Computed and Experimental Binding Enthalpy for Each Force Field

\begin{tabular}{|c|c|c|c|c|c|c|c|c|}
\hline & OPC & SPC & SPC/E & TIP3P & TIP4P & TIP4P-Ew & TIP5P & Bind3P \\
\hline & GAFFv1 & & & & & & & \\
\hline Slope & 0.99 & 1.18 & 1.11 & 1.23 & 1.06 & 1.01 & 0.57 & 1.21 \\
\hline Intercept & 0.42 & 1.22 & -0.24 & 3.20 & -2.55 & -3.69 & -9.40 & 4.76 \\
\hline $\mathbf{R}^{2}$ & 0.88 & 0.93 & 0.89 & 0.93 & 0.86 & 0.86 & 0.38 & 0.93 \\
\hline Kendall's T & 0.80 & 0.85 & 0.80 & 0.82 & 0.78 & 0.78 & 0.36 & 0.79 \\
\hline RMSE & 2.07 & 2.22 & 2.60 & 2.22 & 4.02 & 4.32 & 6.30 & 3.15 \\
\hline MSE & 4.28 & 4.92 & 6.78 & 4.93 & 16.15 & 18.67 & 39.7 & 9.89 \\
\hline \multirow[t]{2}{*}{ MAE } & 1.52 & 1.89 & 2.25 & 1.90 & 3.71 & 3.86 & 5.31 & 2.65 \\
\hline & GAFFv2 & & & & & & & \\
\hline Slope & 1.30 & 1.43 & 1.37 & 1.46 & 1.41 & 1.35 & 0.84 & 1.42 \\
\hline Intercept & 3.86 & 3.96 & 2.14 & 5.01 & 0.47 & 0.28 & -7.93 & 7.91 \\
\hline $\mathbf{R}^{2}$ & 0.61 & 0.79 & 0.79 & 0.80 & 0.70 & 0.69 & 0.45 & 0.79 \\
\hline Kendall's T & 0.55 & 0.61 & 0.62 & 0.64 & 0.59 & 0.56 & 0.33 & 0.59 \\
\hline RMSE & 5.75 & 4.73 & 4.81 & 4.62 & 6.94 & 6.52 & 7.86 & 5.46 \\
\hline MSE & 33.10 & 22.36 & 23.16 & 21.34 & 48.17 & 42.57 & 61.83 & 29.84 \\
\hline \multirow[t]{2}{*}{ MAE } & 4.27 & 4.04 & 4.09 & 4.05 & 6.14 & 5.83 & 6.75 & 4.40 \\
\hline & CGenFF & & & & & & & \\
\hline Slope & 0.09 & 0.35 & 0.26 & 0.40 & 0.32 & 0.21 & -0.09 & 0.45 \\
\hline Intercept & -13.56 & -10.37 & -13.26 & -7.57 & -13.28 & -14.63 & 43.01 & -5.74 \\
\hline $\mathbf{R}^{2}$ & 0.03 & 0.43 & 0.24 & 0.49 & 0.28 & 0.12 & 0.01 & 0.54 \\
\hline Kendall's T & 0.10 & 0.45 & 0.35 & 0.54 & 0.37 & 0.20 & -0.06 & 0.54 \\
\hline RMSE & 6.32 & 4.95 & 6.51 & 3.94 & 6.98 & 7.48 & 56.25 & 3.76 \\
\hline MSE & 39.92 & 24.52 & 42.38 & 15.49 & 48.68 & 55.97 & $3,163.95$ & 14.17 \\
\hline \multirow[t]{2}{*}{ MAE } & 5.23 & 4.27 & 5.38 & 3.45 & 5.83 & 5.98 & 55.8 & 3.22 \\
\hline & Parsley & & & & & & & \\
\hline Slope & 0.53 & 0.76 & 0.70 & 0.81 & 0.72 & 0.58 & 0.11 & 0.83 \\
\hline Intercept & -8.86 & -6.85 & -9.79 & -6.52 & -10.62 & -11.67 & -21.57 & -3.09 \\
\hline $\mathbf{R}^{2}$ & 0.53 & 0.73 & 0.67 & 0.79 & 0.67 & 0.55 & 0.02 & 0.81 \\
\hline Kendall's T & 0.56 & 0.68 & 0.68 & 0.71 & 0.68 & 0.60 & 0.10 & 0.71 \\
\hline RMSE & 4.94 & 4.92 & 6.96 & 4.92 & 7.98 & 7.59 & 12.98 & 2.56 \\
\hline MSE & 24.41 & 24.17 & 48.51 & 24.25 & 63.65 & 57.68 & 168.44 & 6.56 \\
\hline \multirow[t]{2}{*}{ MAE } & 4.02 & 4.08 & 6.24 & 4.26 & 7.34 & 6.69 & 11.21 & 1.83 \\
\hline & SwissParam & & & & & & & \\
\hline Slope & 0.62 & 0.76 & 0.69 & 0.77 & 0.76 & 0.68 & 0.39 & 0.79 \\
\hline Intercept & -9.96 & -8.07 & -10.43 & -3.24 & -11.22 & -12.92 & -13.23 & -0.49 \\
\hline $\mathbf{R}^{2}$ & 0.38 & 0.54 & 0.48 & 0.61 & 0.51 & 0.52 & 0.12 & 0.61 \\
\hline Kendall's T & 0.43 & 0.51 & 0.50 & 0.51 & 0.50 & 0.47 & 0.22 & 0.51 \\
\hline RMSE & 7.25 & 6.58 & 7.95 & 3.59 & 9.37 & 9.97 & 8.95 & 4.08 \\
\hline MSE & 52.50 & 43.35 & 63.19 & 12.86 & 87.85 & 99.37 & 80.03 & 16.63 \\
\hline MAE & 6.27 & 6.07 & 7.27 & 2.68 & 8.85 & 9.19 & 7.04 & 2.86 \\
\hline
\end{tabular}

$\mathrm{R}^{2}$ is the correlation coefficient of determination. RMSE is the root-mean-square error. MSE is the mean squared error. MAE is the mean absolute error. 


\section{Overall Statistics}

Overall, the force field combinations produced a diverse agreement with the experimental enthalpy values (Table 2, and Figure 2). We may define the agreement with experimental values in two ways: i) accuracy, and ii) ranking ability. Here, we report the accuracy of the calculation using slope/intercept, RMSE, MSE, and MAE. The ranking ability is reported by the coefficient of determination $\left(R^{2}\right)$ and Kendall's rank correlation coefficient $(\mathrm{T})$. The smallest RMSE value 2.07 $\mathrm{kca} / \mathrm{mol}$ ), relative to experiment, is obtained with the GAFFv1-OPC combination. Yin et al. ${ }^{30}$ also reported that the OPC water model outperformed Bind3P, TIP3P, TIP4P-Ew, and TIP4P-D water models in binding enthalpy calculations for octa acid and guests parameterized by GAFFv1. In another work by the same research group, TIP4P-Ew produced more accurate results compared to TIP3P, SPC and OPC water models for binding enthalpies of the hosts ( $\alpha$ - and $\beta$-cyclodextrin) and guests. ${ }^{19}$ In the first study, GAFFv1 was used for the parametrization of the host molecule, while the Q4MD-CD force field ${ }^{68}$ was preferred to generate proper conformations matching experimental data of the host molecule, illustrating that careful treatment of all components should always be considered. The largest RMSE, of $56.25 \mathrm{kcal} / \mathrm{mol}$, was observed for CGenFF-TIP5P. No single combination is superior to the others for the binding free enthalpy. However, GAFFv1 may be a preferable default force-field choice for cucurbit[7]uril, since GAFFv1 with all water models, except Bind3P, outperforms all other force-fields. Previous works showed that Bind3P with GAFFv1 produced better results than TIP3P in the binding enthalpy calculations for $\alpha$ - and $\beta$ cyclodextrin, octa acid, and TEMOA. ${ }^{30,69}$

Overall, the Parsley force-field in combination with Bind3P gives the most accurate result (RMSE $=2.56 \mathrm{kcal} / \mathrm{mol}$ ). Parsley is a better force field in terms of reproducing QM conformer energies and geometries than both GAFF versions ${ }^{70}$, but it only produces better enthalpy predictions when used in conjunction with the Bind3P water model. Generally, TIP3P and Bind3P have more accurate results (average RMSE $=\sim 3.8 \mathrm{kcal} / \mathrm{mol}$ ) compared to other water models, and also show a good correlation among all force-fields, especially in GAFFv1. Surprisingly, GAFFv1 produces better results than GAFFv2 despite the fact that GAFFv2 was released to improve the performance and to cover a broader chemical space. ${ }^{71}$ GAFFv1 and GAFFv2 are in the same force fields and most likely tend to be more consistent with each other. However, GAFFv1 is slightly more successful for reproducing QM conformer energies and geometries than GAFFv2. ${ }^{70}$ Also, a problematic "over-definition" of the dihedrals in the GAFFv2 has been identified by a SAMPL7 study. ${ }^{72}$ TIP5P gives the worst results regardless of force-fields. It produces very inaccurate enthalpy values with CGenFF (Figure 2), making this combination one of the worst 
ones we tested. SwissParam (average RMSE $=\sim 6.97 \mathrm{kcal} / \mathrm{mol}$ ) is the worst force-field if TIP5P is ignored because of the result in CGenFF. Binding enthalpies computed with GAFFv1 in combination with TIP3P, Bind3P and SPC water models (Table 2) correlate very well with experiment (Figure 2): linear regression analysis yields a good correlation coefficient $R^{2}=0.93$. On the other hand, CGenFF gives the worst correlations for all water models. Overall, there is a tendency to overestimate the binding enthalpies. The CGenFF server produces default host parameters which clearly require further optimization, at least in our hands. Both GAFFv1 and CGenFF can provide reasonable force fields for reproducing experimental data even though CGenFF performs worse overall in the binding enthalpy calculations. ${ }^{73-74}$ 
A

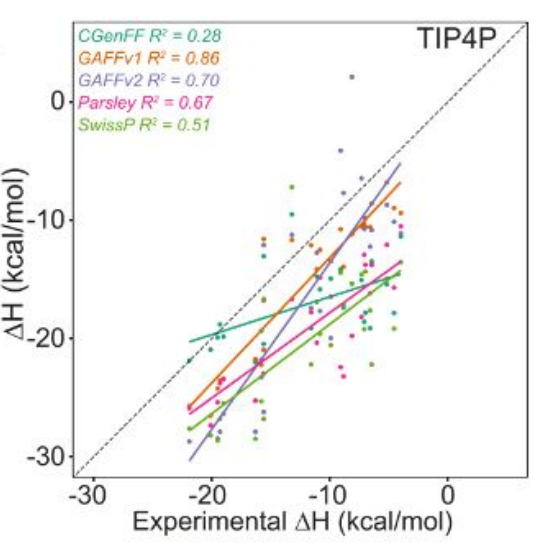

C

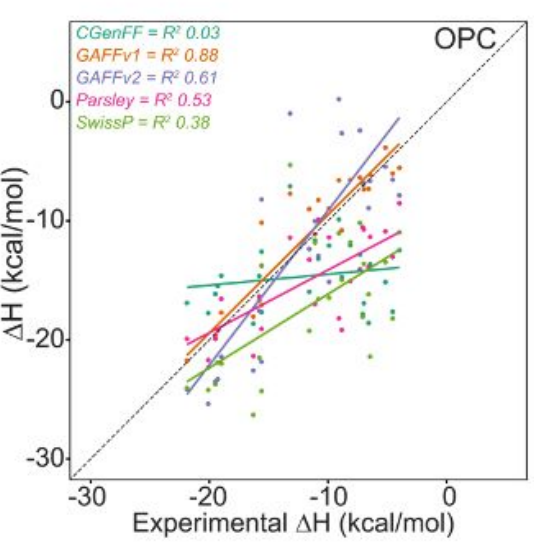

E

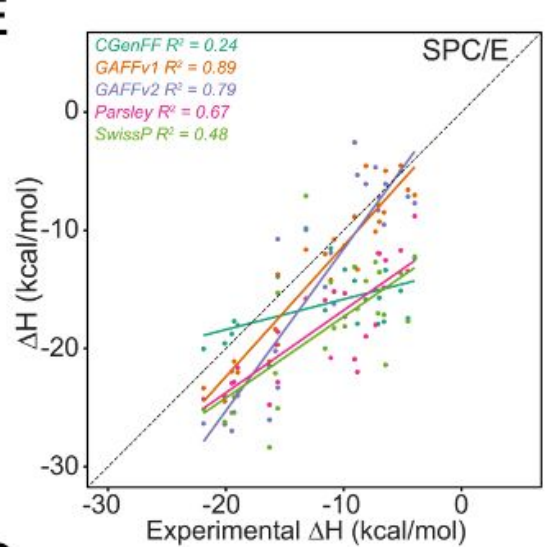

G

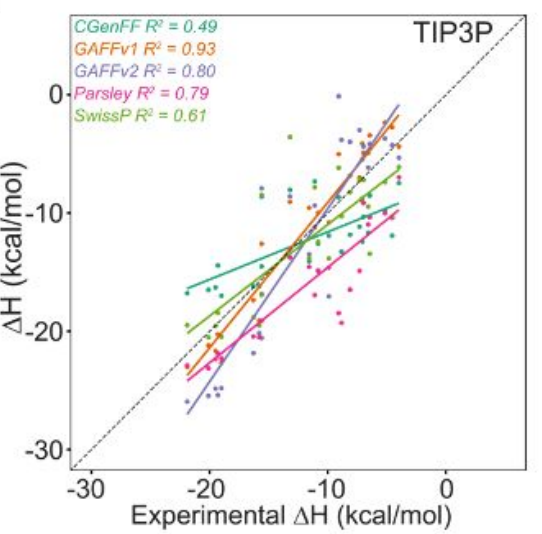

B

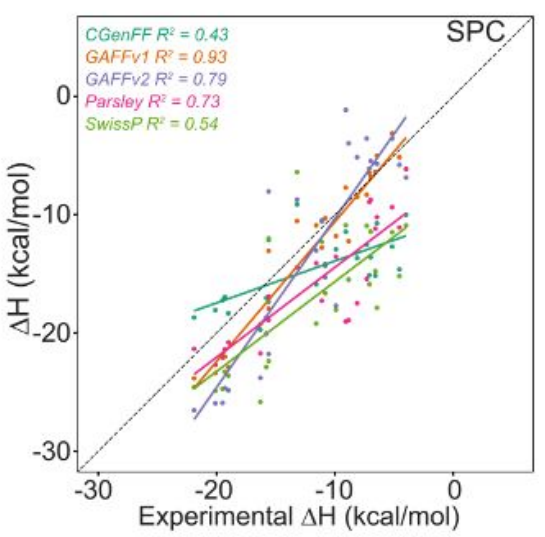

D

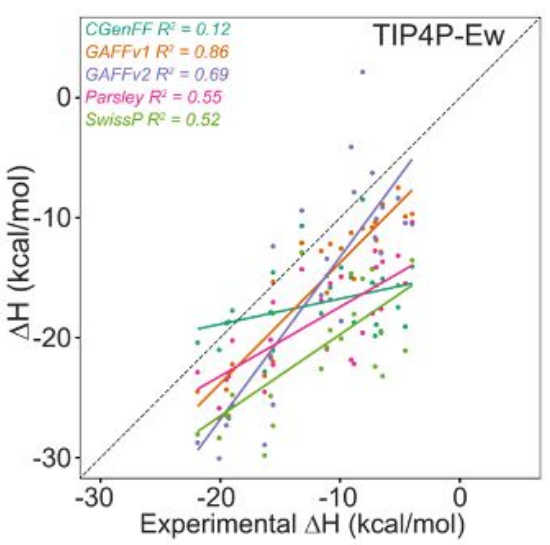

F

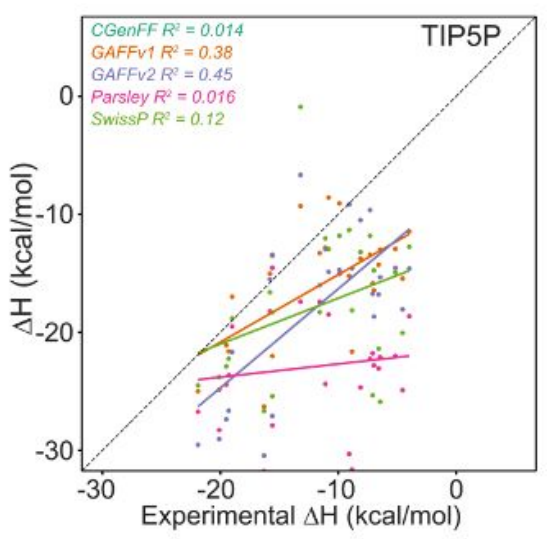

$\mathrm{H}$

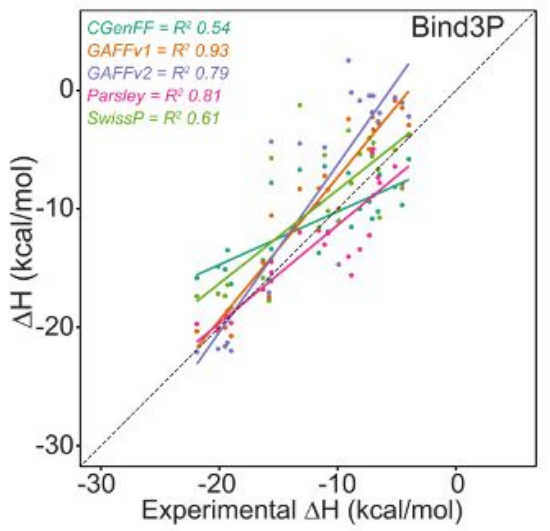


Figure 2. Comparison of calculated binding enthalpies with experimental values for different water models. (A) TIP4P, (B) SPC, (C) OPC, (D) TIP4P-Ew, (E) SPC/E, (F) TIP5P, (G) TIP3P and (H) Bind3P for five different force fields: CGenFF (teal), GAFFv1 (orange), GAFFv2 (purple), Parsley (pink) and SwissParam (lime green). The $\mathrm{R}^{2}$ values are also indicated.

In this assessment, it is also important to consider the uncertainties associated with both the experimental and computational values. The reported experimental uncertainties of the mean range between 0.01 and $2.31 \mathrm{kcal} / \mathrm{mol}$ and most of them are lower than $0.50 \mathrm{kcal} / \mathrm{mol}$. The uncertainties of the calculated binding enthalpies, based on blocking analysis, have a range of 0.40 to $0.89 \mathrm{kcal} / \mathrm{mol}$. Given these uncertainties, it would seem unlikely that the differences between experiment and computation can be attributed to under-sampling and there must be other reasons for the discrepancies. This is supported by the extremely flat convergence graphs shown in Figure 3, suggesting that the simulation trajectories have good conformational sampling.

A

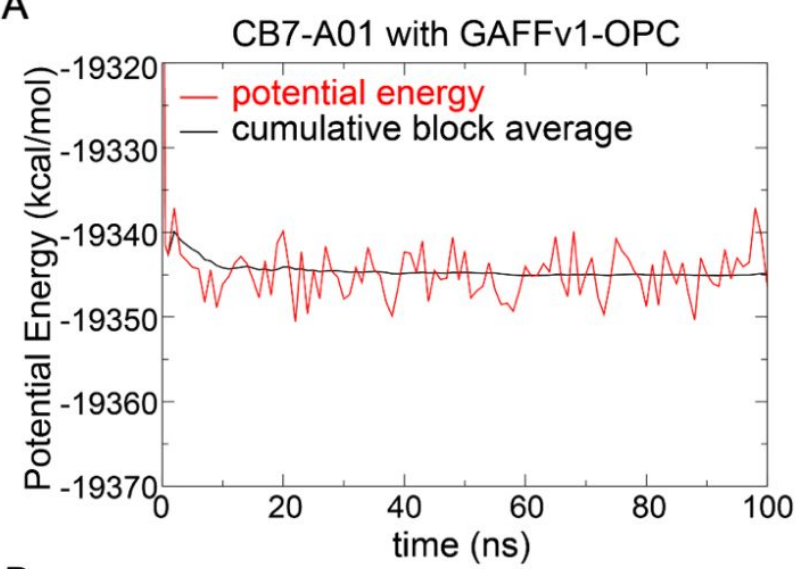

B

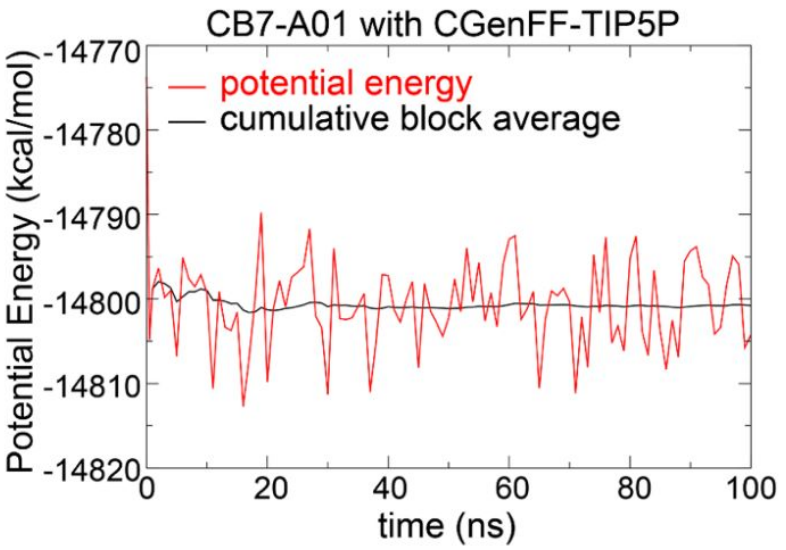

Figure 3. Convergence plot of the binding enthalpy for the systems CB7-A01. The most and worst accurate combination of force-field are included here. Instantaneous potential energy (at every $1 \mathrm{~ns}$ ) is shown in red while the black line shows the cumulative convergence in increasing blocks of $1 \mathrm{~ns}$. 


\section{Impact of the Force-fields on the Host Molecule}

Strategies for force-field development depend on covering chemical space, training data, and the approach to optimizing parameters. ${ }^{75-76}$ Given the challenge of force-field development, there is often huge variability in their performance across a wide change of thermodynamic properties. As part of our efforts to understand the differences in force-fields to predict enthalpy changes, we examined the conformation of the host in simulations of the apo state. Parsley and CGenFF result in more structural deviation than other force-fields (Figure 4), leading to conformations that exhibit a clear distortion away from the regular circular conformation observed in the crystal. It is important to note that this kind of distortion has been observed for $C B 7$ when in complex with guests ${ }^{77}$. Parsley produced the highest structural fluctuation with $1 \pm 0.1 \AA$ and GAFFv2 had the lowest one with $0.30 \pm 0.10 \AA$ (Figure 4).

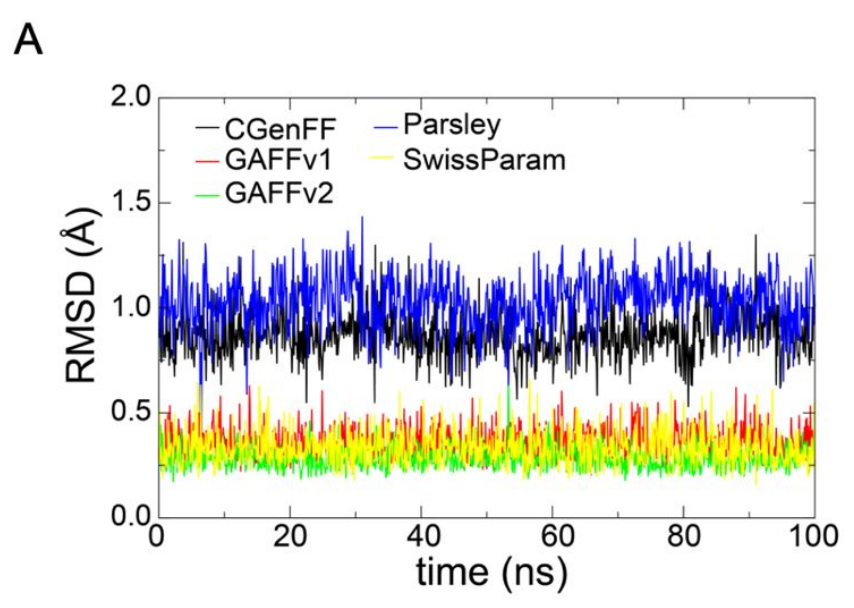

B
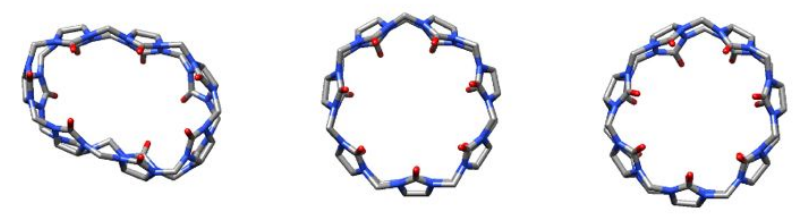

CGenFF

GAFFv1

GAFFv2
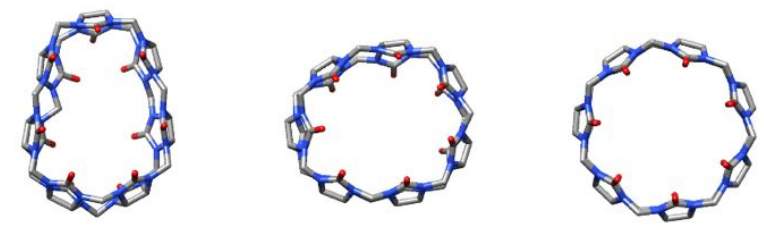

Parsley

SwissParam

PDB: QQ7

Figure 4: (A) RMSD of heavy atoms in the unbound state of the host molecule though the simulations solvated with TIP3P for all force-fields. (B) Final frames of the unbound state of the host solvated by TIP3P and the crystal structure (PDB: QQ7). 


\begin{abstract}
Parsley, also known as the Open Force Field, is the most recent of the force-fields examined here, and was designed to overcome some known limitations of older force-fields, including for example the calculation of hydration free energies and partition coefficients. ${ }^{43,65,78}$ CGenFF was initially developed based on the CHARMM biomolecular force-fields, and it covers a wide range of chemical groups and drug-like molecules. ${ }^{79}$ Another force-field generation tool, SwissParam, generates topologies and parameters derived from MMFF, compatible with the CHARMM forcefields, but SwissParam has been developed for rapid calculations, i.e., docking, minimization, etc. Thus, it may not be suitable for long MD simulations, despite being used in many MD studies. ${ }^{44}$, 80-81 Accordingly, it is useful to include this comparison here. Lastly, we have included GAFFv1 and its second generation, GAFFv2, compatible with the AMBER force-fields. These two forcefields introduce similar dynamics patterns to the host molecule, but the performance is not the same for the enthalpy calculation. Indeed, the presence of the large distortions observed for CGenFF and Parsley does not seem correlated to the performance of enthalpy prediction.
\end{abstract}

The observations of the potential flexibility of CB7 prompted us to assess enthalpy predictions on a host predicted to have a larger range of conformational fluctuation. The acyclic cucurbiturilderived TrimerTrip clip molecule is more flexible compared to our CB7 host molecule (Figure 5). In this analysis, we randomly picked a guest molecule (heptane-1,7-diamine) having a known experimental binding enthalpy value. ${ }^{39} \mathrm{MD}$ simulations using only the TIP3P water model were performed since there is no obvious effect of water models on the host dynamics and TIP3P is considered as a "default" water model. All simulations nicely converged the potential energy illustrated by Figure 5B. GAFFv1 and GAFFv2 successfully computed the binding enthalpy very near to the experimental value $(-10.10 \pm 0.04 \mathrm{kcal} / \mathrm{mol})$, but the performance of CGenFF was relatively poor. SwissParam and Parsley both underestimated the binding enthalpies (Table 3). The pattern of conformational flexibility observed for CB7 where Parsley and CGenFF have more structural deviation compared to the others (Figure 4) was not observed for this system (Figure 5B). 
A

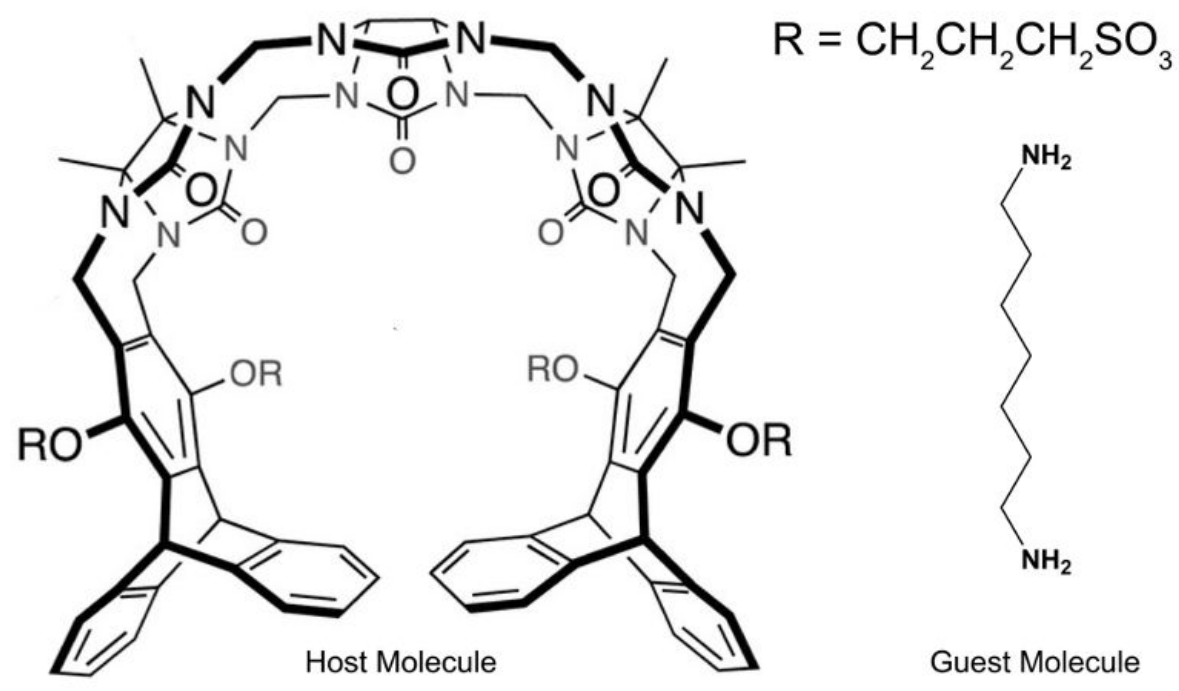

B
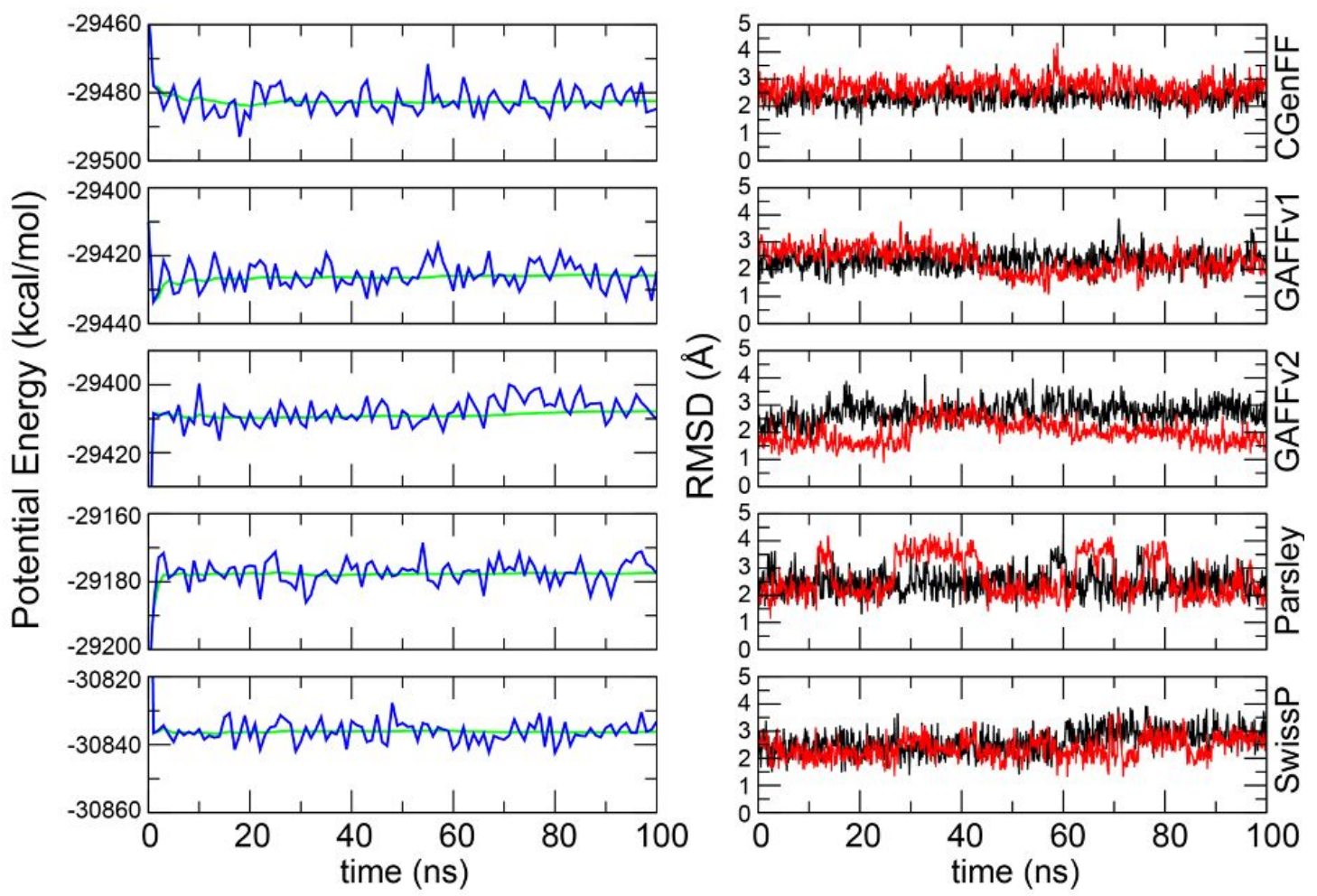

Figure 5: (A) Structure of TrimerTrip and the guest molecule (B) Convergence (green) and change (blue) of the potential energy for the complex systems and RMSD of heavy atoms in the unbound (black) and bound (red) state of the host molecule, TrimerTrip, though the simulations solvated with TIP3P for all force-fields. 
Table 3: Binding enthalpies $(\Delta \mathrm{H}, \mathrm{kcal} / \mathrm{mol})$ for TrimerTrip and the heptane-1,7-diamine guest molecule and the TIP3P water model.

\begin{tabular}{lc}
\hline Method & $\begin{array}{c}\text { Enthalpy } \\
\text { (kcal/mol) }\end{array}$ \\
\hline$\Delta \mathbf{H}_{\text {Exp. }}$ & $-10.10 \pm 0.04$ \\
CgenFF & $-1.10 \pm 0.60$ \\
GAFFv1 & $-9.83 \pm 0.63$ \\
GAFFv2 & $-9.36 \pm 0.62$ \\
Parsley & $-7.22 \pm 0.63$ \\
SwissParam & $-5.57 \pm 0.60$ \\
\hline
\end{tabular}

There are two other tools for topology and parameter generation that we additionally considered in this study. One of them is LigParGen ${ }^{82}$, which was designed to provide OPLS-AA parameters to be used directly in popular MD machines. ${ }^{83}$ We obtained all topologies and parameters for our molecules in our benchmark using a locally-implemented version of the LigParGen software, since the maximum ligand size allowed is 200 atoms on the server. Unfortunately, all host-guest pairs did not stay together during the simulations when these topologies and parameters were used. Further investigation revealed that during the host parametrization process, the tool assigns a normal amide carbon (atom type CAM) to the carbon atoms bonded to the oxygen in the host molecule. This over-simplification may be responsible for the behavior in these simulations, but confirmation of that would require more detailed investigation beyond the scope of this work. Thus, at this stage, we could not report any results from this tool for the OPLS-AA force field. Another tool is the Automated force field Topology Builder (ATB) ${ }^{84}$, which provides topologies and parameters for a wide range of molecules compatible with the GROMOS force-fields. This server performs a multistep process containing a series of quantum mechanical (QM) calculations combined with a knowledge-based approach. ${ }^{84}$ This server can also provide topologies and parameters files that can be read by GROMACS MD engine. However, these simulations did not run to successful conclusion, suggesting the out-of-box results requiring manual intervention, something we were keen to avoid in this assessment. Furthermore the GROMOS force-fields are no longer supported within GROMACS suggesting more other issues pertaining to this forcefield. 85 


\section{The Performance of the Water Models}

Water has many unique and unusual properties and is thus probably the most extensively studied molecule. ${ }^{25,86}$ Our understanding of this tiny molecule is still not complete because of its many extraordinary properties. ${ }^{87-88}$ The complexity of its properties has led to the design of many theoretical and computational models. ${ }^{22,} 89$ In biomolecular simulations, a simulator should carefully select the water model with the most appropriate force-field. ${ }^{28}$ because it is not always obvious whether the water model is suitable under the simulation conditions. Assessment studies of the models to replicate experimental data are crucial to help in the selection of the best water model, but such studies are relatively few.

TIP3P and its derivative Bind3P give the most accurate results compared to other water models, although it must be remembered that different water models may be appropriate for different uses and it is extremely difficult for any one model to reproduce all of the unique properties of water. ${ }^{90}$ A sensitivity analysis approach was used to optimize the Lennard-Jones parameters of the original TIP3P for the development of the Bind3P water model. ${ }^{30}$ Bind3P gives the most accurate result (ie smallest error) when used in conjunction with the Parsley force-field. However, perhaps surprisingly, the best correlation is found when it is used in conjunction with GAFFv1. TIP3P is the best water model when working with GAFFv1. The OPC water model fairs well when used with GAFFv1 $($ RMSE $=2.07 \mathrm{kcal} / \mathrm{mol}$ ). TIP5P is generally a very poor performer regardless of host force-field choice.

\section{The Impact of Aromatic versus Aliphatic Guests on the Assessment}

The guest molecules except CTE, FTE and NTE can be sub-grouped into aromatic and aliphatic, with both groups having eleven guest molecules. We re-analysed the performance of all forcefield and water models according to these groups (SI Raw Data). All water models tend to produce more accurate and correlated results in aliphatic guest molecules than aromatics ones. Bind3P produces the best results with a RMSE $=2.11 \pm 0.41 \mathrm{kcal} / \mathrm{mol}$ and an $\mathrm{R}^{2}=0.82 \pm 0.06$ averaged across all force-fields, while it gives an average RMSE $=3.91 \pm 0.65 \mathrm{kcal} / \mathrm{mol}$ and an $R^{2}=0.30 \pm$ 0.10 for aromatic molecules. GAFFv1 is the best choice for both groups. GAFFv2 has good ranking ability for aliphatic molecules even though it produces more accurate results for aromatic guests with a RMSE $=5.26 \pm 0.67 \mathrm{kcal} / \mathrm{mol}$. The Parsley-Bind3P is the best combination for aliphatic guest molecules with a RMSE of $1.06 \mathrm{kcal} / \mathrm{mol}$, whereas the GAFFv1-OPC combination gives the most accurate result with a RMSE of $1.37 \mathrm{kcal} / \mathrm{mol}$ for aromatic molecules. The most correlated combinations are TIP3P $\left(R^{2}=0.95\right)$ and SPCE $\left(R^{2}=0.72\right)$ with Parsley in aliphatic and 
aromatic molecules, respectively. Interestingly, CGenFF produces more accurate result in aliphatic guests than SwissParam. However, SwissParam's ranking ability prevails over CGenFF. Overall this analysis suggests that there is still a lot of room for improvement in the treatment of aromatic molecules with respect to the calculation of enthalpy.

\section{CONCLUSION}

In this work, the binding enthalpy of 25 guest molecules with CB7 are computed by performing MD simulations using 40 different force-field and water models combinations. The computed binding enthalpies using the TIP3P and its derivative, Bind3P, are with good agreement with the experimental values. GAFFv1, perhaps surprisingly, outperforms the other force-fields, and it produces more accurate results compared to other force-fields. Generally, all force-fields produce better results for aliphatic than aromatic guests suggesting there is scope for improvement in the treatment of aromatic molecules. In these and similar studies, we were not able to include commercially modified force-fields. It would be extremely useful to find ways to assess these as well in our efforts to improve force-field performance. Nevertheless, the findings here should still be useful in ongoing attempts to improve force-fields and how well they reproduce the thermodynamic properties of binding events.

\section{Author Information \\ Corresponding Authors \\ Philip.biggin@bioch.ox.ac.uk \\ ORCIDs \\ Philip Biggin: ORCID 0000-0001-5100-8836}

\section{Acknowledgement}

We thank Dr Irfan Alibay for useful discussions. Computing was supported via Advanced Research Computing (Oxford), the ARCHER UK National Supercomputing Service and JADE (EP/ P020275/1) granted via the High-End Computing Consortium for Biomolecular Simulation, (HECBioSim - http://www.hecbiosim.ac.uk), supported by EPSRC (EP/L000253/1). 


\section{Notes}

The authors declare no competing financial interest.

\section{Supporting Information}

An excel spreadsheet of all data is provided.

A zipped archive of all the parameter and coordinate files used is provided.

\section{References}

(1) Chavent, M.; Lévy, B.; Krone, M.; Bidmon, K.; Nominé, J.-P.; Ertl, T.; Baaden, M., GPUpowered tools boost molecular visualization. Brief. Bioinf. 2011, 12 (6), 689-701.

(2) Stone, J. E.; Hardy, D. J.; Ufimtsev, I. S.; Schulten, K., GPU-accelerated molecular modeling coming of age. J. Mol. Graph. Mod. 2010, 29 (2), 116-125.

(3) Mobley, D. L.; Gilson, M. K., Predicting binding free energies: Frontiers and benchmarks. Ann. Rev. Biophys. 2017, 46 (1), 531-558.

(4) Aldeghi, M.; Heifetz, A.; Bodkin, M. J.; Knapp, S.; Biggin, P. C., Accurate calculation of the absolute free energy of binding for drug molecules. Chem. Sci. 2016, 7, 207-218.

(5) Fogolari, F.; Corazza, A.; Esposito, G., Free energy, enthalpy and entropy from implicit solvent end-point simulations. Front. Mol. Biosci. 2018, 5 (11).

(6) Tang, Z.; Chang, C.-e. A., Binding thermodynamics and kinetics calculations using chemical host and guest: A comprehensive picture of molecular recognition. J. Chem. Theory Comp. 2018, 14 (1), 303-318.

(7) Pan, A.; Kar, T.; Rakshit, A. K.; Moulik, S. P., Enthalpy-entropy compensation (EEC) effect: Decisive role of free energy. J. Phys. Chem. B 2016, 120 (40), 10531-10539.

(8) Dragan, A. I.; Read, C. M.; Crane-Robinson, C., Enthalpy-entropy compensation: the role of solvation. Eur. Biophys. J. 2017, 46 (4), 301-308.

(9) Fox, J. M.; Zhao, M.; Fink, M. J.; Kang, K.; Whitesides, G. M., The Molecular Origin of Enthalpy/Entropy Compensation in Biomolecular Recognition. Annual Review of Biophysics 2018, 47 (1), 223-250.

(10) Schönbeck, C.; Holm, R., Exploring the origins of enthalpy-entropy compensation by calorimetric studies of cyclodextrin complexes. J. Phys. Chem. B 2019.

(11) Han, K.; Hudson, P. S.; Jones, M. R.; Nishikawa, N.; Tofoleanu, F.; Brooks, B. R., Prediction of $\mathrm{CB}[8]$ host-guest binding free energies in SAMPL6 using the double-decoupling method. J. Comput Aided Mol. Des. 2018, 32 (10), 1059-1073.

(12) Bell, D. R.; Qi, R.; Jing, Z.; Xiang, J. Y.; Mejias, C.; Schnieders, M. J.; Ponder, J. W.; Ren, $P$., Calculating binding free energies of host-guest systems using the AMOEBA polarizable force field. Phys. Chem. Chem. Phys. 2016, 18 (44), 30261-30269.

(13) Yin, J.; Henriksen, N. M.; Slochower, D. R.; Shirts, M. R.; Chiu, M. W.; Mobley, D. L.; Gilson, M. K., Overview of the SAMPL5 host-guest challenge: Are we doing better? J. Comput. Aided Mol. Des. 2017, 31 (1), 1-19.

(14) Wickstrom, L.; Deng, N.; He, P.; Mentes, A.; Nguyen, C.; Gilson, M. K.; Kurtzman, T.; Gallicchio, E.; Levy, R. M., Parameterization of an effective potential for protein-ligand binding from host-guest affinity data. J. Mol. Recognit. 2016, 29 (1), 10-21. 
(15) Moghaddam, S.; Inoue, Y.; Gilson, M. K., Host-guest complexes with protein-ligand-like affinities: Computational analysis and design. J. Am. Chem. Soc. 2009, 131 (11), 40124021.

(16) Houk, K. N.; Leach, A. G.; Kim, S. P.; Zhang, X., Binding affinities of host-guest, proteinligand, and protein-transition-state complexes. Angew. Chem. Int. Ed. 2003, 42 (40), 48724897.

(17) Li, A.; Gilson, M. K., Protein-ligand binding enthalpies from near-millisecond simulations: Analysis of a preorganization paradox. J. Chem. Phys. 2018, 149 (7), 072311.

(18) Henriksen, N. M.; Fenley, A. T.; Gilson, M. K., Computational calorimetry: High-precision calculation of host-guest binding thermodynamics. J.Chem. Theory Comput. 2015, 11 (9), 4377-4394.

(19) Henriksen, N. M.; Gilson, M. K., Evaluating force field performance in thermodynamic calculations of cyclodextrin host-guest binding: Water models, partial charges, and host force field parameters. J. Chem. Theory Comput. 2017, 13 (9), 4253-4269.

(20) Fan, S.; lorga, B. I.; Beckstein, O., Prediction of octanol-water partition coefficients for the SAMPL6-logP molecules using molecular dynamics simulations with OPLS-AA, AMBER and CHARMM force fields. J Computer-Aided Mol Des 2020, 34 (5), 543-560.

(21) Best, R. B., Atomistic force fields for proteins. In Biomolecular Simulations: Methods and Protocols, Bonomi, M.; Camilloni, C., Eds. Springer New York: New York, NY, 2019; pp 319.

(22) Guillot, B., A reappraisal of what we have learnt during three decades of computer simulations on water. Journal of Molecular Liquids 2002, 101 (1), 219-260.

(23) Bauer, B. A.; Patel, S., Properties of water along the liquid-vapor coexistence curve via molecular dynamics simulations using the polarizable TIP4P-QDP-LJ water model. J. Chem. Phys. 2009, 131 (8), 084709.

(24) Sakamaki, R.; Sum, A. K.; Narumi, T.; Yasuoka, K., Molecular dynamics simulations of vapor/liquid coexistence using the nonpolarizable water models. J. Chem. Phys. 2011, 134 (12), 124708.

(25) Vega, C.; de Miguel, E., Surface tension of the most popular models of water by using the test-area simulation method. J. Chem. Phys. 2007, 126 (15), 154707.

(26) Montero de Hijes, P.; Sanz, E.; Joly, L.; Valeriani, C.; Caupin, F., Viscosity and self-diffusion of supercooled and stretched water from molecular dynamics simulations. J. Chem. Phys. 2018, 149 (9), 094503.

(27) Lynch, C. I.; Rao, S.; Sansom, M. S. P., Water in Nanopores and Biological Channels: A Molecular Simulation Perspective. Chemical Reviews 2020, 120 (18), 10298-10335.

(28) Nutt, D. R.; Smith, J. C., Molecular dynamics simulations of proteins: Can the explicit water model be varied? J. Chem. Theory Comp. 2007, 3 (4), 1550-1560.

(29) Gao, K.; Yin, J.; Henriksen, N. M.; Fenley, A. T.; Gilson, M. K., Binding enthalpy calculations for a neutral host-guest pair yield widely divergent salt effects across water models. $J$. Chem. Theory Comput. 2015, 11 (10), 4555-4564.

(30) Yin, J.; Henriksen, N. M.; Muddana, H. S.; Gilson, M. K., Bind3P: Optimization of a water model based on host-guest binding data. J. Chem. Theory Comput. 2018, 14 (7), 36213632.

(31) Fenley, A. T.; Henriksen, N. M.; Muddana, H. S.; Gilson, M. K., Bridging calorimetry and simulation through precise calculations of cucurbituril-guest binding enthalpies. J. Chem. Theor. Comput. 2014, 10 (9), 4069-4078.

(32) Erdős, M.; Frangou, M.; Vlugt, T. J. H.; Moultos, O. A., Diffusivity of $\alpha$-, $\beta$-, $\gamma$-cyclodextrin and the inclusion complex of $\beta$-cyclodextrin: Ibuprofen in aqueous solutions; $A$ molecular dynamics simulation study. Fluid Phase Equilib. 2021, 528, 112842.

(33) Moghaddam, S.; Yang, C.; Rekharsky, M.; Ko, Y. H.; Kim, K.; Inoue, Y.; Gilson, M. K., New ultrahigh affinity host-guest complexes of cucurbit[7]uril with bicyclo[2.2.2]octane and 
adamantane guests: Thermodynamic analysis and evaluation of $\mathrm{m} 2$ affinity calculations. $J$. Am. Chem. Soc. 2011, 133 (10), 3570-3581.

(34) Yin, H.; Wang, R.; Wan, J.; Zheng, Y.; Ouyang, D.; Wang, R., Molecular encapsulation of histamine h2-receptor antagonists by cucurbit[7]uril: An experimental and computational study. Molecules 2016, 21, 1178-1189.

(35) Tcyrulnikov, N. A.; Varadharajan, R.; Tikhomirova, A. A.; Pattabiraman, M.; Ramamurthy, V.; Wilson, R. M., Modulation of reduction potentials of bis(pyridinium)alkane dications through encapsulation within cucurbit[7]uril. J. Org. Chem. 2019, 84 (13), 8759-8765.

(36) Yu, J.-S.; Wu, F.-G.; Tao, L.-F.; Luo, J.-J.; Yu, Z.-W., Mechanism of the fast exchange between bound and free guests in cucurbit[7]uril-guest systems. Phys. Chem. Chem. Phys. 2011, 13 (9), 3638-3641.

(37) Miskolczy, Z.; Biczók, L., Kinetics and thermodynamics of berberine inclusion in cucurbit[7]uril. J. Phys. Chem. B 2014, 118 (9), 2499-2505.

(38) Miskolczy, Z.; Megyesi, M.; Toke, O.; Biczók, L., Change of the kinetics of inclusion in cucurbit[7]uril upon hydrogenation and methylation of palmatine. Phys. Chem. Chem. Phys. 2019, 21 (9), 4912-4919.

(39) Ndendjio, S. Z.; Liu, W.; Yvanez, N.; Meng, Z.; Zavalij, P. Y.; Isaacs, L., Triptycene walled glycoluril trimer: synthesis and recognition properties. New J. Chem. 2020, 44 (2), 338-345.

(40) Vanommeslaeghe, K.; Raman, E. P.; MacKerell, A. D., Automation of the CHARMM general force field (CGenFF) II: Assignment of bonded parameters and partial atomic charges. J. Chem. Inf. Model. 2012, 52 (12), 3155-3168.

(41) Vanommeslaeghe, K.; MacKerell, A. D., Automation of the charmm general force field (CGenFF) I: Bond perception and atom typing. J. Chem. Inf. Model. 2012, 52 (12), 31443154.

(42) Wang, J.; Wolf, R. M.; Caldwell, J. W.; Kollman, P. A.; Case, D. A., Development and testing of a general amber force field. J. Comp. Chem. 2004, 25 (9), 1157-1174.

(43) Qiu, Y.; Smith, D.; Boothroyd, S.; Jang, H.; Wagner, J.; Bannan, C.; Gokey, T.; Lim, V.; Stern, C.; Rizzi, A.; Lucas, X.; Tjanaka, B.; Shirts, M.; Gilson, M.; Chodera, J.; Bayly, C.; Mobley, D.; Wang, L.-P., Development and benchmarking of open force field v1.0.0, the parsley small molecule force field. ChemRxiv: 2020.

(44) Zoete, V.; Cuendet, M. A.; Grosdidier, A.; Michielin, O., SwissParam: A fast force field generation tool for small organic molecules. J. Comp. Chem. 2011, 32 (11), 2359-2368.

(45) Izadi, S.; Anandakrishnan, R.; Onufriev, A. V., Building water models: A different approach. J. Phys. Chem. Letts. 2014, 5 (21), 3863-3871.

(46) Robinson, G. W., 1996; Vol. 9.

(47) Kusalik, P. G.; Svishchev, I. M., The spatial structure in liquid water. Science 1994, 265 (5176), 1219.

(48) Jorgensen, W. L.; Chandresekhar, J.; Madura, J. D.; Impey, R. W.; Klein, M. L., Comparison of simple potential functions for simulating liquid water. J. Chem. Phys. 1983, 79, 926-935.

(49) Horn, H. W.; Swope, W. C.; Pitera, J. W.; Madura, J. D.; Dick, T. J.; Hura, G. L.; HeadGordon, T., Development of an improved four-site water model for biomolecular simulations: TIP4P-Ew. J. Chem. Phys. 2004, 120 (20), 9665-9678.

(50) Wang, J.; Wang, W.; Kollman, P. A.; Case, D. A., Antechamber: An accessory software package for molecular mechanical calculations. J. Am. Chem. Soc 2001, 222, U403.

(51) Jakalian, A.; Jack, D. B.; Bayly, C. I., Fast, efficient generation of high-quality atomic charges. AM1-BCC model: II. Parameterization and validation. J. Comput. Chem. 2002, 23 (16), 1623-1641.

(52) Jakalian, A.; Bush, B. L.; Jack, D. B.; Bayly, C. I., Fast, efficient generation of high-quality atomic charges. AM1-BCC model: I. Method. . J. Comput. Chem. 2000, 21, 132-146.

(53) Vanommeslaeghe, K.; MacKerell Jr, A. D. The CHARMM General Force Field (CGenFF) interface. https://cgenff.umaryland.edu/ (accessed Jan 20, 2021). 
(54) Zoete, V.; Cuendet, M.; Grosdidier, A.; Michielin, O., SwissParam: A fast force field generation tool for small organic molecules. J. Comput. Chem. 2011, 32, 2359-2368.

(55) Zoete, V.; Cuendet, M. A.; Grosdidier, A.; Michielin, O. SwissParam. https://www.swissparam.ch/ (accessed 20th Jan 2021).

(56) Abraham, M. J.; Murtola, T.; Schulz, R.; Páll, S.; Smith, J. C.; Hess, B.; Lindahl, E., GROMACS: High performance molecular simulations through multi-level parallelism from laptops to supercomputers. SoftwareX 2015, 1-2, 19-25.

(57) Van Der Spoel, D.; Lindahl, E.; Hess, B.; Groenhof, G.; Mark, A. E.; Berendsen, H. J., GROMACS: fast, flexible, and free. J Comput Chem 2005, 26 (16), 1701-18.

(58) Berendsen, H. J. C.; van der Spoel, D.; van Drunen, R., GROMACS: A message-passing parallel molecular dynamics implementation. Comp. Phys. Comm. 1995, 95, 43-56.

(59) Ponder, J. W.; Richards, F. M., An efficient newton-like method for molecular mechanics energy minimization of large molecules. J. Comp. Chem. 1987, 8 (7), 1016-1024.

(60) Bussi, G.; Donadio, D.; Parrinello, M., Canonical sampling through velocity rescaling. J. Chem. Phys. 2007, 126.

(61) Parrinello, M.; Rahman, A., Polymorphic transitions in single crystals: A new molecular dynamics method. J. Appl. Phys. 1981, 52 (12), 7182-7190.

(62) Hess, B., P-lincs: A parallel linear constraint solver for molecular simulation. J. Chem. Theor. Comput. 2008, 4 (1), 116-122.

(63) Essman, U.; Perera, L.; Berkowitz, M. L.; Darden, T.; Lee, H.; Pedersen, L. G., A smooth particle mesh Ewald method. J. Chem. Phys. 1995, 103, 8577-8593.

(64) Lee, R. M.; Conduit, G. J.; Nemec, N.; López Ríos, P.; Drummond, N. D., Strategies for improving the efficiency of quantum Monte Carlo calculations. Physical Review E 2011, 83 (6), 066706.

(65) Wolff, U., Monte Carlo errors with less errors. computer physics communications 2004, 156 (2), 143-153.

(66) Flyvbjerg, H.; Petersen, H. G., Error estimates on averages of correlated data. The Journal of Chemical Physics 1989, 91 (1), 461-466.

(67) Spencer, J. pyblock, http://github.com/jsspencer/pyblock.

(68) Cézard, C.; Trivelli, X.; Aubry, F.; Djedaïni-Pilard, F.; Dupradeau, F.-Y., Molecular dynamics studies of native and substituted cyclodextrins in different media: 1. Charge derivation and force field performances. Phys. Chem. Chem. Phys. 2011, 13 (33), 15103-15121.

(69) Erdős, M.; Hartkamp, R.; Vlugt, T. J. H.; Moultos, O. A., Inclusion complexation of organic micropollutants with $\beta$-cyclodextrin. J. Phys. Chem. B 2020, 124 (7), 1218-1228.

(70) Lim, V. T.; Hahn, D. F.; Tresadern, G.; Bayly, C. I.; Mobley, D. M., Benchmark assessment of molecular geometries and energies from small molecule force fields. F1000 Research 2020, 9, 1390.

(71) Träg, J.; Zahn, D., Improved GAFF2 parameters for fluorinated alkanes and mixed hydroand fluorocarbons. J. Mol. Model. 2019, 25 (2), 39.

(72) Khalak, Y.; Tresadern, G.; de Groot, B. L.; Gapsys, V., Non-equilibrium approach for binding free energies in cyclodextrins in SAMPL7: force fields and software. J. Comput-Aid. Mol. Des. 2020.

(73) Zhu, S., Validation of the generalized force fields GAFF, CGenFF, OPLS-AA, and PRODRGFF by testing against experimental osmotic coefficient data for small drug-like molecules. J. Chem. Inf. Mod. 2019, 59 (10), 4239-4247.

(74) Kumar, V.; Rane, K. S.; Wierzchowski, S.; Shaik, M.; Errington, J. R., Evaluation of the performance of GAFF and CGenFF in the prediction of liquid-vapor saturation properties of naphthalene derivatives. Ind. Eng. Chem. Res. 2014, 53 (41), 16072-16081.

(75) Dauber-Osguthorpe, P.; Hagler, A. T., Biomolecular force fields: where have we been, where are we now, where do we need to go and how do we get there? J. Comput. Aided Mol. Des. 2019, 33 (2), 133-203. 
(76) Nerenberg, P. S.; Head-Gordon, T., New developments in force fields for biomolecular simulations. Curr. Opin. Struct. Biol. 2018, 49, 129-138.

(77) Mei, L.; Xie, Z.-N.; Hu, K.-q.; Yuan, L.-Y.; Gao, Z.-Q.; Chai, Z.-F.; Shi, W.-Q., Supramolecular host-guest inclusion for distinguishing cucurbit[7]uril-based pseudorotaxanes from smallmolecule ligands in coordination assembly with a uranyl center. Chemistry - Eur. J. 2017, 23 (56), 13995-14003.

(78) Kenney, I. M.; Beckstein, O.; lorga, B. I., Prediction of cyclohexane-water distribution coefficients for the SAMPL5 data set using molecular dynamics simulations with the OPLSAA force field. J. Comput-Aid. Mol. Des. 2016, 30 (11), 1045-1058.

(79) Vanommeslaeghe, K.; Hatcher, E.; Acharya, C.; Kundu, S.; Zhong, S.; Shim, J.; Darian, E.; Guvench, O.; Lopes, P.; Vorobyov, I.; Mackerell Jr, A. D., CHARMM general force field: A force field for drug-like molecules compatible with the CHARMM all-atom additive biological force fields. J Comput Chem 2010, 31 (4), 671-690.

(80) Rao, P.; Shukla, A.; Parmar, P.; Rawal, R. M.; Patel, B.; Saraf, M.; Goswami, D., Reckoning a fungal metabolite, Pyranonigrin A as a potential Main protease (Mpro) inhibitor of novel SARS-CoV-2 virus identified using docking and molecular dynamics simulation. Biophys. Chem. 2020, 264, 106425.

(81) Jiang, X.; Yang, K.; Yuan, B.; Gong, B.; Wan, L.; Patil, N. A.; Swarbrick, J. D.; Roberts, K. D.; Schreiber, F.; Wang, L.; Velkov, T.; Li, J., Simulations of octapeptin-outer membrane interactions reveal conformational flexibility is linked to antimicrobial potency. J. Biol. Chem. 2020, 295 (47), 15902-15912.

(82) Dodda, L. S.; Cabeza de Vaca, I.; Tirado-Rives, J.; Jorgensen, W. L., LigParGen web server: An automatic OPLS-AA parameter generator for organic ligands. . Nucleic Acids Res. 2017, 45, W331-W336.

(83) Dodda, L. S.; Cabeza de Vaca, I.; Tirado-Rives, J.; Jorgensen, W. L., LigParGen web server: an automatic OPLS-AA parameter generator for organic ligands. Nucleic Acids Res. 2017, 45 (W1), W331-W336.

(84) Malde, A. K.; Zuo, L.; Breeze, M.; Stroet, M.; Poger, D.; Nair, P. C.; Oostenbrink, C.; Mark, A. E., An Automated Force Field Topology Builder (ATB) and Repository: Version 1.0. J. Chem. Theory Comput. 2011, 7 (12), 4026-4037.

(85) Hess, B.; van der Spoel, D.; Abraham, M. J.; Lindahl, E., On the importance of accurate algorithms for reliable molecular dynamics simulations. ChemRXiv 2019, preprint.

(86) Kale, S.; Herzfeld, J., Natural polarizability and flexibility via explicit valency: The case of water. J. Chem. Phys. 2012, 136 (8), 084109.

(87) Finney, J. L., The water molecule and its interactions: the interaction between theory, modelling, and experiment. Journal of Molecular Liquids 2001, 90 (1), 303-312.

(88) Daniel, R. M.; Finney, J. L.; Stoneham, M.; Finney, J. L., Water? What's so special about it? Philosophical transactions of the royal society of london. Series b: Biological sciences 2004, 359 (1448), 1145-1165.

(89) Ouyang, J. F.; Bettens, R. P. A., Modelling water: A lifetime enigma. Chima Int. J. Chem. 2015, 69 (3), 104-111.

(90) Mark, P.; Nilsson, L., Structure and dynamics of the TIP3P, SPC, and SPC/E water models at 298 K. J. Phys. Chem. A 2001, 105 (43), 9954-9960. 
TOC

$\Delta \mathrm{H}: 8$ water models with 5 force fields
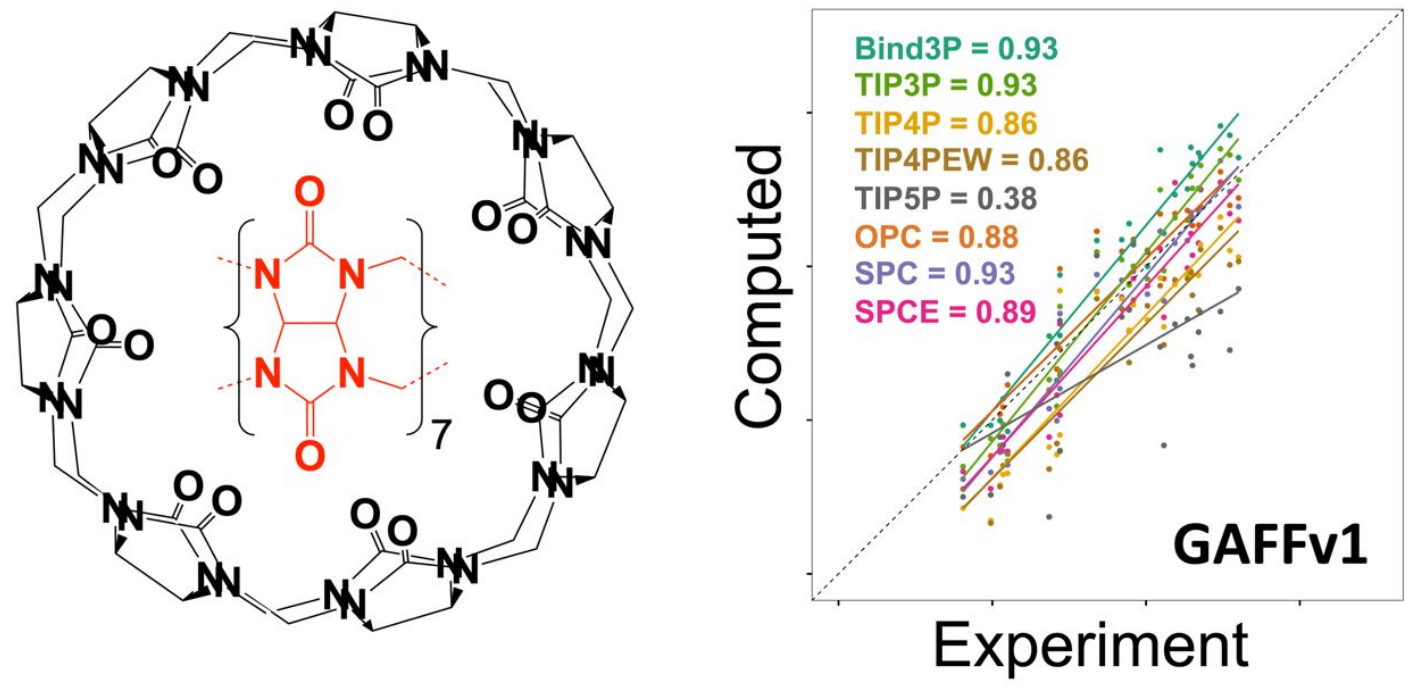